International Journal of Pure and Applied Mathematics

Volume 102 No. 3 2015, 533-545

ISSN: 1311-8080 (printed version); ISSN: 1314-3395 (on-line version)

url: http://www.ijpam.eu

doi: http://dx.doi.org/10.12732/ijpam.v102i3.11

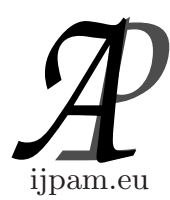

\title{
ON A QUANTUM SYSTEM EXPOSED TO A HEAT BATH
}

\author{
Eugen Grycko ${ }^{1}$, Werner Kirsch ${ }^{2}$, Tobias Mühlenbruch ${ }^{3}$ \\ ${ }^{1,2,3}$ Department of Mathematics and Computer Science \\ University of Hagen \\ Universitätsstrasse 1 \\ D-58084 Hagen, GERMANY
}

\begin{abstract}
An appropriate generator matrix induces in a natural way a Markov semigroup which can be applied to the construction of a continuous time Markov process whose asymptotic distribution is independent of the initial state. We propose a generator associated with a prescribed (arbitrary) distribution which turns out to be reversible for the corresponding Markov process. As an application, we show that the Markov process offers a physically plausible description of a quantum system exposed to a heat bath. The system approaches its Gibbs state corresponding to the temperature of the heat bath if its initial state commutes with the Hamiltonian.
\end{abstract}

AMS Subject Classification: 82C10, 60J27

Key Words: fixed point theorem, Doob's theorem, homogeneous Markov process

\section{Introduction}

A classical question known from Thermodynamics concerns the process of approaching a thermal equilibrium by a physical system. Usually the identification of the equilibrium (Gibbs) state is motivated by the entropy principle. The mathematical rationalization of the convergence of a quantum system to the Gibbs state is often hard to carry out.

Received: May 8, 2015

(c) 2015 Academic Publications, Ltd.

${ }^{\S}$ Correspondence author url: www.acadpubl.eu 
Recently quantum systems described by Hamiltonians acting on a finitely dimensional Hilbert space have drawn some attention. They are attractive from the computational viewpoint and offer explanations for some quantum phenomena, cf. [4] and [5].

In the present contribution we assume that a Hamiltonian acts on $\mathbb{C}^{N}$ entailing that the discussion of the corresponding Gibbs state can be based essentially on Linear Algebra. A stochastic Markov model describing the behavior of a quantum system exposed to a heat bath is proposed. This model abstracts of the details of the particular thermal contact and involves the temperature as the only parameter characterizing the heat bath. The convergence of the constructed Markov process to the Gibbs state of the quantum system is essentially implied by a fixed point argument.

\section{On a Markov Process Induced by a Generator Matrix}

Let us consider the finite set $S:=\{1, \ldots, N\}$ where $N$ is a positive integer. We endow $S$ with the $\sigma$-algebra $\mathcal{P}(S)$ (power set of $S$ ). For $j, k \in S, j \neq k$, let $a_{j k}>0$ be a positive real and put

$$
a_{j j}:=-\sum_{k \neq j} a_{j k} \quad(j \in S) .
$$

We utilize matrix $A:=\left(a_{j k}\right)_{j, k=1}^{N}$ as a generator of a semigroup $(B(t))_{t \geq 0}$ of linear operators

$$
B(t):=\exp (t \cdot A) \quad(t \geq 0)
$$

We observe that

$$
B(s) B(t)=B(s+t) \quad(s, t \geq 0)
$$

holds; moreover,

$$
B(t)=I_{N}+t A+O\left(t^{2}\right)
$$

for $t \searrow 0$ where $O$ and $I_{N}$ denotes the Landau symbol and the $N \times N$-identity matrix, respectively.

Let $t>0$ be arbitrary. For a sufficiently large integer $n$ all entries $B_{j k}(t / n)$ in $B(t / n)$ are strictly positive (cf. (2.4)); since (2.3) implies the identity

$$
B(t)=(B(t / n))^{n},
$$

we have

$$
B_{j k}(t)>0 \quad(j, k \in S ; t>0) .
$$


Let $e=(1, \ldots, 1)^{\prime} \in \mathbb{R}^{N}$ be the column vector of ones. Because of the validity of

$$
B(0) e=I_{N} e=e
$$

and of

$$
\frac{d}{d t} B(t) e=\exp (t A) A e=0 \quad(t \geq 0)
$$

(cf. (2.1)), it follows that

$$
B(t) e=e \quad(t \geq 0),
$$

which means that $B(t)$ is a stochastic matrix whose rows sum up to 1 for $t \geq 0$. Therefore $(B(t))_{t \geq 0}$ can be interpreted as a Markov semigroup.

Let $q=\left(q_{1}, \ldots, q_{N}\right) \in \mathbb{R}_{+}^{N}$ be such a row vector that

$$
\sum_{j=1}^{N} q_{j}=1
$$

holds. $q$ can be interpreted as a probability measure (initial distribution) on $(S, \mathcal{P}(S))$. According to [1], Theorem 36.4 and Theorem 35.3, $q$ and $(B(t))_{t \geq 0}$ induce a probability measure $P$ (say) on the set $S^{\mathbb{R}_{+}}$of functions $\omega: \mathbb{R}_{+} \rightarrow S$ endowed with the product $\sigma$-algebra $\mathcal{P}(S)^{\mathbb{R}_{+}}$. The random variable

$$
\pi_{t}: S^{\mathbb{R}_{+}} \rightarrow S, \quad \pi_{t}(\omega):=\omega(t)
$$

is distributed according to the probability measure $q B(t)$ in the sense of

$$
\left(P\left(\pi_{t}=1\right), \ldots, P\left(\pi_{t}=N\right)\right)=q B(t)
$$

for $t \geq 0$. $\left(\pi_{t}\right)_{t \geq 0}$ is a homogeneous Markov process whose marginal distributions are given by $q B(t)$ for $t \geq 0$.

The set of probability distributions on $(S, \mathcal{P}(S))$ can be identified with

$$
\Delta_{N}:=\left\{q=\left(q_{1}, \ldots, q_{N}\right) \in \mathbb{R}_{+}^{N} \mid \sum_{j=1}^{N} q_{j}=1\right\} .
$$

Let us introduce the 1-norm on $\mathbb{R}^{N}$ :

$$
|x|_{1}:=\sum_{j=1}^{N}\left|x_{j}\right| \quad\left(x=\left(x_{1}, \ldots, x_{n}\right) \in \mathbb{R}^{N}\right) .
$$


$|\cdot|_{1}$ induces the metric

$$
d: \mathbb{R}^{N} \times \mathbb{R}^{N} \rightarrow \mathbb{R}_{+}, \quad d(x, y):=|x-y|_{1} .
$$

Now, $(B(t))_{t \geq 0}$ is interpreted as a semigroup of maps

$$
B(t): \Delta_{N} \rightarrow \Delta_{N}, \quad q \mapsto q B(t),
$$

for $t \geq 0$.

For $q^{(1)}, q^{(2)} \in \Delta_{N}, q^{(1)} \neq q^{(2)}$, we have

$$
\begin{aligned}
d\left(q^{(1)} B(t), q^{(2)} B(t)\right) & =\sum_{k=1}^{N}\left|\sum_{j=1}^{N}\left(q_{j}^{(1)}-q_{j}^{(2)}\right) B_{j k}(t)\right| \\
& <\sum_{j=1}^{N} \sum_{k=1}^{N}\left|q_{j}^{(1)}-q_{j}^{(2)}\right| B_{j k}(t) \\
& =\sum_{j=1}^{N}\left|q_{j}^{(1)}-q_{j}^{(2)}\right| \cdot \sum_{k=1}^{N} B_{j k}(t) \\
& =\sum_{j=1}^{N}\left|q_{j}^{(1)}-q_{j}^{(2)}\right| \\
& =d\left(q^{(1)}, q^{(2)}\right)
\end{aligned}
$$

for $t>0$ (cf. (2.5) and (2.6)), which means that $B(t): \Delta_{N} \rightarrow \Delta_{N}, q \mapsto q B(t)$, is a contractive map. Since $\Delta_{N}$ is compact, a fixed point theorem of Banach type (cf. [2],[3]) implies that there exists an unique fixed point $\bar{q} \in \Delta_{N}$ of $B(t): \Delta_{N} \rightarrow \Delta_{N}$ for $t>0$ and that

$$
\lim _{t \rightarrow \infty}\left(P\left(\pi_{t}=1\right), \ldots, P\left(\pi_{t}=N\right)\right)=\lim _{t \rightarrow \infty} q B(t)=\bar{q}
$$

holds for every initial distribution $q \in \Delta_{N}$. Moreover, the process $\left(\pi_{t}\right)_{t \geq 0}$ is stationary if and only if the initial distribution $q$ is equal to $\bar{q}$.

We endow $S$ with the discrete metric $\delta: S \times S \rightarrow \mathbb{R}_{+}, \delta(j, k):=\delta_{j k}$, where $\delta_{j k}$ denotes the Kronecker symbol; $\delta$ induces the Borel $\sigma$-algebra $\mathcal{P}(S)$ on $S$.

We formulate and prove the following

Lemma 2.1. Let $q \in \Delta_{N}$ and $(B(t))_{t>0}$ be a Markov semigroup defined according to (2.2). Let $P$ be the probability measure on $\left(S^{\mathbb{R}+}, \mathcal{P}(S)^{\mathbb{R}_{+}}\right)$satisfying (2.8) where $\left(\pi_{t}\right)_{t \geq 0}$ is the Markov process introduced in (2.7). Let $\left(t_{l}\right)_{l=0}^{\infty}$ be such a sequence in $\mathbb{R}_{+}$that

$$
\lim _{l \rightarrow \infty} t_{l}=t_{0}
$$


holds. Then

$$
\lim _{l \rightarrow \infty} P\left(\delta\left(\pi_{t_{l}}, \pi_{t_{0}}\right) \geq \varepsilon\right)=0 \quad(\varepsilon>0),
$$

which means that the process $\left(\pi_{t}\right)_{t \geq 0}$ is continuous in probability at $t_{0}$.

Proof. Fix $0<\varepsilon<1$. (2.4) implies that

$$
\begin{aligned}
P\left(\delta\left(\pi_{t_{l}}, \pi_{t_{0}}\right)>\varepsilon\right) & =\sum_{j \neq k} P\left(\pi_{t_{l}}=j \text { and } \pi_{t_{0}}=k\right) \\
& \leq \sum_{j \neq k}\left(\left|t_{l}-t_{0}\right| \cdot a_{j k}+O\left(\left|t_{l}-t_{0}\right|^{2}\right)\right) \rightarrow 0
\end{aligned}
$$

holds for $l \rightarrow \infty$.

Remark 2.2. A typical trajectory of the process $\left(\pi_{t}\right)_{t \geq 0}$ jumps between the states $j \in S$.

Let $M \subset S^{\mathbb{R}_{+}}$denote the set of functions $\omega: \mathbb{R}_{+} \rightarrow S$ that are $\mathcal{B}\left(\mathbb{R}_{+}\right)-\mathcal{P}(S)$ measurable where $\mathcal{B}\left(\mathbb{R}_{+}\right)$denotes the Borel $\sigma$-algebra on $\mathbb{R}_{+}$. We endow $M$ with the trace $\sigma$-algebra

$$
\mathcal{M}:=\left\{F \cap M \mid F \in \mathcal{P}(S)^{\mathbb{R}_{+}}\right\} .
$$

Let $F \in \mathcal{P}(S)^{\mathbb{R}_{+}}$be a set such that $F \supset M$. There exists a countable set $C \subset \mathbb{R}_{+}$and a measurable set $D \in \mathcal{P}(S)^{C}$ such that

$$
F=\left(\pi_{C}^{\mathbb{R}_{+}}\right)^{-1}(D)
$$

where $\pi_{C}^{\mathbb{R}_{+}}: S^{\mathbb{R}_{+}} \rightarrow S^{C}$ denotes the projection

$$
\pi_{C}^{\mathbb{R}_{+}}(\omega):=(\omega(t))_{t \in C}
$$

Note that $\pi_{C}^{\mathbb{R}_{+}}$is $\mathcal{P}(S)^{\mathbb{R}_{+}}-\mathcal{P}(S)^{C}$ measurable.

Since the $\mathcal{B}\left(\mathbb{R}_{+}\right)-\mathcal{P}(S)$ measurability of a function $\omega: \mathbb{R}_{+} \rightarrow S$ is not affected if its values $\omega(t)$ are arbitrarily chosen for $t$ from the countable set $C$, it follows that

$$
\begin{aligned}
F & =\left(\pi_{C}^{\mathbb{R}_{+}}\right)^{-1}\left(\pi_{C}^{\mathbb{R}_{+}}(F)\right)=\left(\pi_{C}^{\mathbb{R}_{+}}\right)^{-1}(D) \\
& \supset\left(\pi_{C}^{\mathbb{R}_{+}}\right)^{-1}\left(\pi_{C}^{\mathbb{R}_{+}}(M)\right)=\left(\pi_{C}^{\mathbb{R}_{+}}\right)^{-1}\left(S^{C}\right)=S^{\mathbb{R}_{+}}
\end{aligned}
$$


holds.

We have in particular shown that

$$
P^{*}(M):=\inf \left\{P(F) \mid F \in \mathcal{P}(S)^{\mathbb{R}_{+}} \text {and } F \supset M\right\}=1
$$

holds where $P^{*}$ denotes the outer measure of $P$ on $\left(S^{\mathbb{R}_{+}}, \mathcal{P}(S)^{\mathbb{R}_{+}}\right)$. Doob's theorem (cf. [1], Theorem 38.2) implies now that $M$ can be chosen as the path-set for the Markov process $\left(\pi_{t}\right)_{t \in \mathbb{R}_{+}}$whose finitely dimensional marginal distributions are determined by the initial distribution $q \in \Delta_{N}$ and and the Markov semigroup $(B(t))_{t \geq 0}$, cf. [1], 36 .

\section{A Class of Markov Processes with a Prescribed Reversible Distribution}

Let $\lambda>0$ and $p=\left(p_{1}, \ldots, p_{N}\right) \in \Delta_{N}$ be such that $p_{j}>0$ holds for $j=1, \ldots, N$. Define the generator matrix $A=\left(a_{j k}\right)_{j, k=1}^{N}$ by

$$
a_{j k}:=\lambda \cdot\left(\frac{p_{k}}{p_{j}}\right)^{1 / 2} \quad(j \neq k)
$$

and

$$
a_{j j}:=-\sum_{k \neq j} a_{j k} \quad(j=1, \ldots, N) .
$$

Obviously,

$$
A e=0 .
$$

As in Section 2, we define the Markov semigroup $(B(t))_{t \geq 0}$ by

$$
B(t):=\exp (t A) \quad(t \geq 0)
$$

Let the distribution $p$ be coded by the diagonal matrix $\Pi=\left(\Pi_{j k}\right)_{j, k=1}^{N}$ according to

$$
\Pi_{j k}= \begin{cases}p_{j} & \text { for } j=k \\ 0 & \text { elsewhere }\end{cases}
$$

Standard matrix algebra yields that

$$
\Pi A \Pi^{-1}=A^{\prime}
$$

holds where $A^{\prime}$ denotes the transpose of $A$. It follows that

$$
\Pi B(t)=B^{\prime}(t) \Pi \quad(t \geq 0)
$$


is satisfied where $B^{\prime}(t)$ denotes the transpose of $B(t)$. (3.4) can be rewritten as

$$
p_{j} B_{j k}(t)=p_{k} B_{k j}(t) \quad(j, k=1, \ldots, N ; t \geq 0) .
$$

We say that distribution $p$ is reversible w.r.t. Markov semigroup $(B(t))_{t \geq 0}$. For the $j^{\text {th }}$ component $(p B(t))_{j}$ of the row vector $p B(t)$ we have

$$
(p B(t))_{j}=\sum_{k=1}^{N} p_{k} B_{k j}(t)=\sum_{k=1}^{N} p_{j} B_{j k}(t)=p_{j}
$$

for $j=1, \ldots, N$ and $t \geq 0$. This means that $p$ is the unique fixed point of the contractive map

$$
B(t): \Delta_{N} \rightarrow \Delta_{N}, \quad q \mapsto q B(t),
$$

for $t>0$. Again (cf. Section 2),

$$
\lim _{t \rightarrow \infty} q B(t)=\lim _{t \rightarrow \infty}\left(P\left(\pi_{t}=1\right), \ldots, P\left(\pi_{t}=N\right)\right)=p
$$

where $\left(\pi_{t}\right)_{t \geq 0}$ denotes the Markov process induced by the initial distribution $q$ and the Markov semigroup $(B(t))_{t \geq 0}$.

Remark 3.1. If $(B(t))_{t \geq 0}$ represents the dynamics of a physical system whose initial state is described by an arbitrary distribution $q \in \Delta_{N}$, then the system approaches the reversible equilibrium state $p$ for the time parameter $t \rightarrow \infty$, cf. (3.7). In particular the system is "approximatively reversible" for large values of $t$.

\section{Schrödinger Dynamics and the Gibbs State}

Let $H$ be a $N \times N$ Hermitian matrix. We interpret $H$ as a Hamiltonian of a quantum system. Let $Z(0)$ be its initial state. This means that $Z(0)$ is a positive matrix (with complex entries) such that

$$
\operatorname{trace}(Z(0))=1
$$

holds.

If the system is thermally isolated, then it is natural to impose the Schrödinger dynamics on it:

$$
Z(t):=\exp \left(-\frac{i t}{\hbar} \cdot H\right) Z(0) \exp \left(\frac{i t}{\hbar} \cdot H\right)
$$


where $Z(t)$ describes the quantum state at time point $t \geq 0$ and $\hbar$ denotes the Planck constant. Since the matrix

$$
\begin{aligned}
\exp \left(-\frac{i t}{\hbar} \cdot H\right)^{*} & =\exp \left(\frac{i t}{\hbar} \cdot H^{*}\right) \\
& =\exp \left(\frac{i t}{\hbar} \cdot H\right) \\
& =\exp \left(-\frac{i t}{\hbar} \cdot H\right)^{-1}
\end{aligned}
$$

is unitary $\left({ }^{*}\right.$ denotes the transposition and complex conjugation), $Z(t)$ is positive and

$$
\operatorname{trace}(Z(t))=1
$$

holds for $t \geq 0$.

If the initial state commutes with $H$, then (4.1) implies that $Z(0)$ is invariant w.r.t. the Schrödinger dynamics:

$$
Z(t)=Z(0) \quad(t \geq 0) .
$$

Entropic considerations (cf. [6], p. 384) suggest that the thermal equilibrium of a quantum system is described by the Gibbs state

$$
G(\beta):=(\operatorname{trace}(\exp (-\beta H)))^{-1} \cdot \exp (-\beta H)
$$

if the system is exposed to a heat bath whose inverse temperature is denoted by $\beta \geq 0$. Note that $G(\beta)$ is positive, it commutes with $H$ and

$$
\operatorname{trace}(G(\beta))=1
$$

holds.

Let $\Phi=\left(\varphi_{1}, \ldots, \varphi_{N}\right)$ be an orthonormal basis of $\mathbb{C}^{N}$ consisting of eigenvectors $\varphi_{j}$ of $H$ :

$$
H \varphi_{j}=h_{j} \varphi_{j} \quad(j=1, \ldots, N)
$$

where $h_{1} \leq h_{2} \leq \ldots \leq h_{N}$ are the eigenvalues. To avoid the trivial case let us assume that $h_{1}<h_{N}$ holds. Put

$$
\bar{h}:=\frac{1}{N} \cdot \sum_{j=1}^{N} h_{j} .
$$


According to the quantum formalism, the expectation of energy of the system at state $G(\beta)$ is given by

$$
\mathbb{E}_{\beta}(H):=\operatorname{trace}(G(\beta) H)=(\tau(\beta))^{-1} \cdot \sum_{j=1}^{N}\left(\exp \left(-\beta h_{j}\right) h_{j}\right)
$$

where

$$
\tau(\beta):=\sum_{j=1}^{N} \exp \left(-\beta h_{j}\right)=\operatorname{trace}(\exp (-\beta H)) .
$$

Applying Steiner's theorem we obtain for the derivative

$$
\begin{aligned}
\frac{d}{d \beta} \mathbb{E}_{\beta}(H)= & -(\tau(\beta))^{-1} \cdot \sum_{j=1}^{N}\left(\exp \left(-\beta h_{j}\right) h_{j}^{2}\right) \\
& +\left((\tau(\beta))^{-1} \cdot \sum_{j=1}^{N}\left(\exp \left(-\beta h_{j}\right) h_{j}\right)\right. \\
= & -\mathbb{V}_{\beta}(H)<0
\end{aligned}
$$

where $\mathbb{V}_{\beta}(H)$ denotes the variance of $H$ :

$$
\mathbb{V}_{\beta}(H):=\operatorname{trace}\left(G(\beta) \cdot\left(H-\mathbb{E}_{\beta}(H)\right)^{2}\right)=\mathbb{E}_{\beta}\left(H^{2}\right)-\left(\mathbb{E}_{\beta}(H)\right)^{2}>0 .
$$

It follows that the function $\beta \mapsto \mathbb{E}_{\beta}(H)$ is strictly decreasing for $\beta \in \mathbb{R}$. Since

$$
\mathbb{E}_{0}(H)=\bar{h} \quad \text { and } \quad \lim _{\beta \rightarrow \infty} \mathbb{E}_{\beta}(H)=h_{1},
$$

the intermediate value theorem implies that we can assign an unique value of inverse temperature $\beta \in \mathbb{R}_{+}$to every prescribed expectation value $\mathbb{E}_{\beta}(H) \in$ $\left(h_{1}, \bar{h}\right]$ of energy.

Remark 4.1. Although there might be physical objections to allowing for negative $\beta$, we can, as a mathematical speculation, assign an unique $\beta<0$ if an energy value $\mathbb{E}_{\beta}(H) \in\left(\bar{h}, h_{N}\right)$ is required. (Note that

$$
\lim _{\beta \rightarrow-\infty} \mathbb{E}_{\beta}(H)=h_{N}
$$

holds.)

Remark 4.2. Let $d_{j}$ denote the dimension of the eigenspace of $H$ corresponding to the eigenvalue $h_{j}$ for $j=1, \ldots, N$. Let us assume that the system 
has attained the Gibbs state $G(\beta)$ for a $\beta \in \mathbb{R}_{+}$. The probability $p_{j}$ of observing the energy $h_{j}$ is given by

$$
p_{j}=(\tau(\beta))^{-1} \cdot d_{j} \cdot \exp \left(-\beta h_{j}\right) \quad(j=1, \ldots, N) .
$$

Remark 4.3. Let $\psi_{j}: \mathbb{C}^{N} \rightarrow \mathbb{C}^{N}$ denote the orthogonal projection onto the 1-dimensional space spanned by $\varphi_{j}, j=1, \ldots, N$. For $q(0)=\left(q_{1}(0), \ldots, q_{N}(0)\right) \in$ $\Delta_{N}$ the quantum state

$$
Z(0):=\sum_{j=1}^{N} q_{j}(0) \cdot \psi_{j}
$$

commutes with $H$. Conversely, if a quantum state $Z(0)$ commutes with $H$, then it can be represented by (4.7) where an orthonormal basis $\Phi=\left(\varphi_{1}, \ldots, \varphi_{N}\right)$ of eigenvectors and the corresponding projections $\psi_{1}, \ldots, \psi_{N}: \mathbb{C}^{N} \rightarrow \mathbb{C}^{N}$ and a distribution $q(0)=\left(q_{1}(0), \ldots, q_{N}(0)\right) \in \Delta_{N}$ are appropriately selected.

Remark 4.4. Let $\psi_{1}: \mathbb{C}^{N} \rightarrow \mathbb{C}^{N}$ denote the orthogonal projection onto the 1-dimensional space spanned by the eigenvector $\varphi_{1}$ corresponding to the minimal eigenvalue $h_{1}$ of $H$. It follows that for $\beta \rightarrow \infty$ the Gibbs state converges to the ground state:

$$
\lim _{\beta \rightarrow \infty} G(\beta)=\psi_{1}
$$

\section{A Dynamics of a Quantum System Exposed to a Heat Bath}

Let a quantum system be described by Hamiltonian $H$ which is represented by a Hermitian $N \times N$ matrix. We assume that the initial state $Z(0)$ of the system commutes with $H$. It follows that there exists such an orthonormal basis $\Phi=\left(\varphi_{1}, \ldots, \varphi_{N}\right)$ of $\mathbb{C}^{N}$ that the matrices corresponding to operators $H$ and $Z(0)$ w.r.t. $\Phi$ are diagonal with entries

$$
H_{j k}= \begin{cases}h_{j} & \text { for } j=k \\ 0 & \text { elswhere }\end{cases}
$$

and

$$
Z_{j k}(0)= \begin{cases}q_{j}(0) & \text { for } j=k \\ 0 & \text { elsewhere }\end{cases}
$$


where $q(0)=\left(q_{1}(0), \ldots, q_{N}(0)\right) \in \Delta_{N}$ and

$$
h_{1} \leq h_{2} \leq \ldots \leq h_{N}
$$

are the eigenvalues of $H$,

$$
H \varphi_{j}=h_{j} \cdot \varphi_{j} \quad(j=1, \ldots, N),
$$

and the $\varphi_{j}$ are also eigenvectors of $Z(0)$ :

$$
Z(0) \varphi_{j}=q_{j}(0) \cdot \varphi_{j} \quad(j=1, \ldots, N) .
$$

We assume, moreover, that $h_{1}<h_{N}$ holds.

Let us suppose that the system is in a thermal contact with a heat bath of temperature $T>0$; this entails that

$$
\beta=\frac{1}{k_{B} \cdot T}
$$

holds where $\beta>0$ is the inverse temperature and $k_{B}$ denotes the Boltzmann constant. Put

$$
\tau(\beta)=\operatorname{trace}(\exp (-\beta H))=\sum_{j=1}^{N} \exp \left(-\beta h_{j}\right) .
$$

Let the intensity of the thermal contact be expressed by a constant $\lambda>0$ carrying the physical unit $\mathrm{s}^{-1}$. An attractive choice is, for example,

$$
\lambda=\frac{k_{B} \cdot T}{\hbar} .
$$

Analogously to Section 3, we introduce the now physically interpretable generator matrix $A=\left(a_{j k}\right)_{j, k=1}^{N}$ by

$$
a_{j k}:=\lambda \cdot \exp \left(-\frac{\beta}{2} \cdot\left(h_{k}-h_{j}\right)\right) \quad(j \neq k)
$$

and

$$
a_{j j}:=-\lambda \cdot \sum_{k \neq j} \exp \left(-\frac{\beta}{2} \cdot\left(h_{k}-h_{j}\right)\right) \quad(j=1, \ldots, N) .
$$

(5.2) and (5.3) are now special cases of (3.1) and (3.2) where the reversible distribution $p=\left(p_{1}, \ldots, p_{N}\right) \in \Delta_{N}$ considered in Section 3 corresponds to the Gibbs distribution of energy

$$
\left(p_{1}, \ldots, p_{N}\right)=(\tau(\beta))^{-1} \cdot\left(\exp \left(-\beta h_{1}\right), \ldots, \exp \left(-\beta h_{N}\right)\right) .
$$


We interpret

$$
(\tau(\beta))^{-1} \cdot \exp \left(-\beta \cdot h_{j}\right)
$$

as the probability for observing eigenstate $\varphi_{j}$ when the system has attained the quantum Gibbs state $G(\beta)$, cf. (4.4).

Under the assumption that the thermal contact with the heat bath does not affect the commutation relation

$$
Z(t) H=H Z(t)
$$

for $t \geq 0$, it is natural to introduce the Markov semigroup $(B(t))_{t \geq 0}$ according to (3.3). (5.5) and (4.3) suggest that we can neglect the Schrödinger dynamics which may be supposed to act on the system. The initial state $Z(0)$ and semigroup $(B(t))_{t \geq 0}$ induce a Markov process (cf. Section 3) in the state space $S$ whose element $j$ is supposed to code the eigenstate $\varphi_{j}$ of Hamiltonian $H$ and of operators $(Z(t))_{t \geq 0}$ for $j=1, \ldots, N$.

Remark 5.1. The considered quantum system attains the states

$$
q(t)=\left(q_{1}(t), \ldots, q_{N}(t)\right)=q(0) B(t) \in \Delta_{N} \quad(t \geq 0)
$$

and

$$
\lim _{t \rightarrow \infty} q(t)=p
$$

holds. The density operator $Z(t)$ describing the quantum state of the system at time point $t$ has the following representation w.r.t. basis $\Phi$ :

$$
Z_{j k}(t)= \begin{cases}q_{j}(t) & \text { for } j=k \\ 0 & \text { elsewhere }\end{cases}
$$

Remark 5.2. A jump in a trajectory $\omega \in M$ from $j$ to $k$ at a time point $t_{0} \in \mathbb{R}_{+}$is interpreted as an instantaneous exchange of the energy quantum $h_{k}-h_{j}$ between the heat bath and the considered system. If $\Delta E=h_{k}-h_{j}>0$, then the system absorbs the energy $\Delta E$; the case $\Delta E=h_{k}-h_{j}<0$ corresponds to the emission of energy from the quantum system to the heat bath.

Remark 5.3. Modeling a quantum system exposed to a heat bath by the Markov process $\left(\pi_{t}\right)_{t \geq 0}$ entails that the system approaches the Gibbs state associated with Hamiltonian $H$ and the inverse temperature $\beta>0$ for $t \rightarrow \infty$, cf. (3.7),(5.6) and (5.4). 


\section{Acknowledgments}

The authors would like to thank Reinhard Börger, Riccardo Catalano, Winfried Hochstättler, Hans Konrad Knörr, Thomas Müller, Karl-Eugen Spreng and Andreas Wiegner for stimulating discussions concerning the present contribution.

\section{References}

[1] H. Bauer, Probability Theory. De Gruyter, Berlin, New York (1996).

[2] M. Edelstein, On fixed and periodic points under contractive mappings. $J$. Lond. Math. Soc. 37, (1962), 74-79.

[3] E. Grycko, A fixed point theorem with applications to random sequences. Seminarberichte Mathematik 51, Hagen (1995).

[4] E. Grycko, W. Kirsch, T. Mühlenbruch, Some quantum mechanical evidence for the amplification of thermal noise in an electrostatic field. Int. J. Pure Appl. Math. 69, No. 4, (2011), 437-507.

[5] E. Grycko, W. Kirsch, T. Mühlenbruch, Spontaneous amplification of thermal noise by a slack joint. Int. J. Pure Appl. Math. 88, No. 4, (2013), 593-601.

[6] W. Thirring, Quantum Mathematical Physics. Second edition, Springer, Berlin, Heidelberg, New York (2002). 
